\title{
Hadamard products of certain power series
}

\author{
by \\ HABIB SHARIF (Shiraz)
}

1. Introduction. In [8] we defined an E-algebraic function, an analogue of the concept of a differentially algebraic function over a perfect field $K$ of characteristic $p>0$. In this paper, we shall study the Hadamard product of differentially algebraic and E-algebraic power series. The $p$-multiplicative and $p$-additive power series are introduced and it will be shown that these two classes of series are closed under the Hadamard product operation. We also show that the Hadamard product of a rational power series with a D-algebraic (respectively, E-algebraic) power series in several variables is not necessarily D-algebraic (respectively, E-algebraic).

From now on $L$ will denote a field of characteristic zero. Suppose that $P\left(x_{0}, x_{1}, \ldots, x_{n+1}\right)$ is a non-zero polynomial in $n+2$ variables with coefficients in $L$. Then the equation

$$
P\left(x, \omega(x), \omega^{\prime}(x), \ldots, \omega^{(n)}(x)\right)=0,
$$

where $\omega^{(n)}=d \omega^{(n-1)} / d x, \omega^{(0)}(x)=\omega(x)$, is called an algebraic differential equation (ADE, for short). A function (or a formal power series) $f(x) \in$ $L[[x]]$ which satisfies an ADE as above is called differentially algebraic (Dalgebraic, for short) over $L(x)$. In other words, a function $f(x)$ is D-algebraic (over $L(x)$ ) if and only if $f, f^{\prime}, \ldots, f^{(n)}, \ldots$ are algebraically dependent over $L(x)$.

Example 1.1. (i) The series $f(x)=\sum_{n \geq 0} n ! x^{n}$ is D-algebraic since $x^{2} f^{\prime}(x)+(x-1) f(x)+1=0$.

(ii) The series $f=\sum_{n \geq 0} x^{n^{2}}$ is also D-algebraic (see [4]).

A function (or a power series) $f$ is called transcendentally transcendental (TT, for short) if it is not D-algebraic. That is, if it satisfies no algebraic differential equation. For example, the series $g=\sum_{n \geq 0} x^{2^{n}}$ is TT (see [4]).

1991 Mathematics Subject Classification: Primary 11D88; Secondary 11J99. 
REMARKS 1.2. (i) Let $f=\sum_{n=0}^{\infty} a_{n} x^{n} \in L[[x]]$ and $M=L\left(x, f, f^{\prime}, \ldots\right.$ $\ldots, f^{(n)}, \ldots$ ). Then $f$ is a D-algebraic series (over $L(x)$ ) if and only if tr. deg. ${ }_{L(x)} M<\infty$, where tr. deg. ${ }_{K} F$ means the transcendence degree of $F$ over $K$.

(ii) Recall that for a field $K$, an element $f \in K((x))$ is called an algebraic function over $K$ if $f$ is algebraic over the field of rational functions $K(x)$. An algebraic formal power series over the field $L$ of characteristic zero is a series which satisfies a differential equation of zero order and hence a D-algebraic power series. In other words, if $f$ is algebraic, then $\operatorname{tr} \cdot \operatorname{deg} \cdot L(x) M=0$.

A special type of a D-algebraic power series is a differentially finite (Dfinite, for short) power series. Roughly speaking, a D-finite power series is a series which satisfies a linear differential equation. In other words, a power series $f \in L[[x]]$ is said to be D-finite if $f$ together with all its derivatives $f^{(n)}=d^{n} f / d x^{n}, n \geq 1$, span a finite-dimensional subspace of $L((x))$, regarded as a vector space $L(x)$.

The function of Example 1.1(i) is D-finite, while the function $\tan (x)$ is not [10].

2. E-algebraic functions. Since the $p$ th derivative of a power series $f$ over a field of characteristic $p>0$ collapses to zero, the notion of $\mathrm{D}$ algebraicity is not so significant over fields of characteristic $p>0$. In [8] we defined an analogue of the concept of a D-algebraic function over a perfect field $K$ of characteristic $p>0$, as follows. From now on $K$ will denote a perfect field of characteristic $p>0$ and $\mathbb{Z}_{p}$ will denote the ring of $p$-adic integers.

Lemma 2.1. If $f(x) \in K[[x]]$ (respectively $K((x))$ ), then $f$ can be written uniquely as

$$
\sum_{i=0}^{p-1} x^{i} f_{i}^{p}
$$

for some $f_{i} \in K[[x]]$ (respectively $\left.K((x))\right)$.

Proof. See [9]. (Note that $f_{i}=\sum_{n=0}^{\infty} a_{p n+i}^{1 / p} x^{n}$.)

For each $i \in\{0,1, \ldots, p-1\}$ define $E_{i}: K((x)) \rightarrow K((x))$ by $E_{i}(f)=f_{i}$. Now for $f \in K((x))$, we have

$$
f=\sum_{i=0}^{p-1} x^{i}\left[E_{i}(f)\right]^{p} .
$$

The operator $E_{i}$ is semilinear; that is, if $f, g \in K((x))$ and $\lambda \in K$, then

$$
E_{i}(\lambda f+g)=\lambda^{1 / p} E_{i}(f)+E_{i}(g) .
$$

Moreover, $E_{i}\left(g^{p} f\right)=g E_{i}(f)$. 
Let $\Omega$ be the semigroup generated by the identity operator and the $E_{i}$ for $i \in\{0,1, \ldots, p-1\}$ with ordinary composition as multiplication. With each $f \in K((x))$ we associate its orbit $\Omega(f)=\{E(f): E \in \Omega\}$.

Definition. Suppose that $f \in K((x))$. We say that $f$ is an E-algebraic function (over $K(x)$ ) if tr. deg. $K(x) K(x, \Omega(f))<\infty$.

Notation. We shall denote by $\Gamma_{K}$ the set of all E-algebraic functions.

ExAmple 2.2. (i) Any algebraic function over $K$ is E-algebraic.

(ii) Let $K=\mathbb{F}_{2}$ and $\alpha$ be a 2-adic integer. Let $f_{\alpha}=(1+x)^{\alpha} \in \mathbb{F}_{2}[[x]]$. Then $f_{\alpha} \in \Gamma_{K}$. (Note that if $\alpha \notin \mathbb{Q}$, then $f$ is not algebraic; see, for example, $[5,7]$.)

In [8] we showed that $\Gamma_{K}$ with ordinary addition and multiplication of series is a field with some natural properties. Note also that by Example 2.2, we have

$$
\overline{K(x)} \subset \Gamma_{K} \subset K((x))
$$

3. Hadamard products of D-algebraic and E-algebraic power series. Recall that for the power series $f=\sum_{n>0} a_{n} x^{n}$ and $g=\sum_{n>0} b_{n} x^{n}$, the Hadamard product of $f$ and $g$, denoted by $f * g$, is the series defined by $f * g=\sum_{n \geq 0} a_{n} b_{n} x^{n}$.

$D_{L}$, the set of all D-algebraic power series over $L$, is not closed under the Hadamard product operation, as the following example shows.

EXAMPLE 3.1. Let $f=\sum_{n \geq 0} x^{n^{2}}$ and $g=\sum_{n \geq 0}(1 / n !) x^{n}$. Then $f$ and $g$ are D-algebraic. However,

$$
f * g=\sum_{n \geq 0} \frac{1}{\left(n^{2}\right) !} x^{n^{2}},
$$

which is not D-algebraic (see [4]).

An example of a subalgebra of $D_{L}$ which is closed under the Hadamard product operation is the algebra of D-finite power series (see [10]).

Note that in Example 3.1, $g$ is D-finite. Hence the Hadamard product of a D-algebraic and a D-finite power series is not D-algebraic. However, in [6] we proved the following result.

Theorem 3.2. Suppose that $f, g \in L[[x]]$. If $f$ is rational and $g$ is $D$ algebraic, then $f * g$ is D-algebraic.

In analogy with the case of characteristic zero, we shall prove the following theorem.

Theorem 3.3. Suppose that $f, g \in K[[x]]$. If $f$ is rational and $g$ is E-algebraic, then $f * g$ is E-algebraic.

First we need some lemmas. 
Lemma 3.4. Suppose that $M$ is an algebraic extension of $K$. Suppose that $f \in K[[x]]$. If tr. $\operatorname{deg}_{{ }_{M}} M(x, \Omega(f))<\infty$, then $\operatorname{tr} . \operatorname{deg} \cdot K K(x, \Omega(f))<\infty$.

Proof. Since $K \subseteq M$ is an algebraic extension, tr. deg. ${ }_{K} M(x, \Omega(f))$ is finite. Now as $K(x, \Omega(f))$ is a field contained in $M(x, \Omega(f))$ we have tr. deg. $_{K} K(x, \Omega(f))<\infty$.

Lemma 3.5. Suppose that $h(x)=\sum_{n=0}^{\infty} a_{n} x^{n} \in \Gamma_{K}$. Then

$$
h(\beta x)=\sum_{n=0}^{\infty} a_{n} \beta^{n} x^{n} \in \Gamma_{K} \quad \text { for } \beta \in K .
$$

Proof. By Lemma 2.1 we have

$$
\begin{aligned}
h(\beta x) & =\sum_{n=0}^{\infty} a_{n} \beta^{n} x^{n}=\sum_{n=0}^{\infty} \sum_{i=0}^{p-1} a_{p n+i} \beta^{p n+i} x^{p n+i} \\
& =\sum_{i=0}^{p-1} x^{i}\left(\beta^{i / p} \sum_{n=0}^{\infty} a_{p n+i}^{1 / p}(\beta x)^{n}\right)^{p} .
\end{aligned}
$$

Hence $E_{i}(h(\beta x))=\beta^{i / p} E_{i}(h)(\beta x)$ for each $i \in\{0,1, \ldots, p-1\}$ and similarly for "higher order" of $E$. Thus, when changing the variable from $x$ to $\beta x$ in $h$, each element of $\Omega(h(\beta x))$ differs from the corresponding element of $\Omega(h)(\beta x)$ by some power $\beta^{1 / p}$, which is an element of $K$. By the same argument used in the proof of Theorem 2.7 of [6], as $K(x, \Omega(h)(\beta x))=$ $K(x, \Omega(h(\beta x)))$, we have tr. deg. $K K(x, \Omega(h(\beta x)))<\infty$. That is, $h(\beta x) \in$ $\Gamma_{K}$ as required.

Proof of Theorem 3.3. By Lemma 3.4 we can assume that $K$ is algebraically closed. Hence, as $f$ is rational, we can write

$$
f(x)=P(x)+\sum_{i=0}^{N} \frac{\alpha_{i}}{\left(1-\beta_{i} x\right)^{a_{i}}},
$$

where $P(x) \in K[x], \alpha_{i}, \beta_{i} \in K$ and $a_{i} \in \mathbb{N}$.

Since the Hadamard product operation is a $K$-bilinear operation it is enough to prove the theorem for the case $f_{1}=1 /(1-\beta x)^{a}, a \geq 1$, where $\beta \in K$. Let $a^{\prime}, k$ be non-negative integers such that $a+a^{\prime}=p^{k}$ so that

$$
f_{1}=\frac{1}{(1-\beta x)^{a}}=\frac{(1-\beta x)^{a^{\prime}}}{1-\beta^{p^{k}} x^{p^{k}}}=(1-\beta x)^{a^{\prime}} \sum_{n \geq 0} \beta^{p^{k} n} x^{p^{k} n} .
$$

Again by the $K$-bilinearity of the Hadamard product operation it is enough to prove the theorem for the case $f_{2}=\sum_{n \geq 0} \beta^{p^{k} n} x^{p^{k} n+s}$ for $\beta \in K, s \in \mathbb{N}$, 
$0 \leq s \leq p^{k}-1$. Hence we have to show that if $g=\sum_{n \geq 0} b_{n} x^{n}$, then

$$
f_{2} * g=\left(\sum_{n \geq 0} \beta^{p^{k} n} x^{p^{k} n+s}\right) * \sum_{n \geq 0} b_{n} x^{n}=\sum_{n \geq 0} \beta^{p^{k} n} b_{p^{k} n+s} x^{p^{k} n+s} \in \Gamma_{K} .
$$

If we show that $t(x)=\sum_{n \geq 0} b_{p^{k} n+s} x^{p^{k} n+s} \in \Gamma_{K}$, then by Lemma 3.5

$$
\frac{1}{\beta^{s}} t(\beta x)=\sum_{n \geq 0} \beta^{p^{k} n} b_{p^{k} n+s} x^{p^{k} n+s}=f_{2} * g \in \Gamma_{K} .
$$

Note that if $k=0$, then as $g \in \Gamma_{K}$, by Lemma 3.5 we have

$$
f_{2} * g=g(\beta x)=\sum_{n \geq 0} \beta^{n} b_{n} x^{n} \in \Gamma_{K} .
$$

Hence we may assume that $k \geq 1$. Since $s \in\left\{0,1, \ldots, p^{k}-1\right\}$, we can write $s=p^{k-1} s_{1}+\ldots+p s_{k-1}+s_{k}$, where $0 \leq s_{i}<p$, for $i=1, \ldots, k$. Now, as $g \in \Gamma_{K}$, we have

$$
\begin{aligned}
t(x) & =\sum_{n \geq 0} b_{p^{k} n+s} x^{p^{k} n+s}=x^{s}\left[\sum_{n \geq 0} b_{p\left(p\left(\ldots p\left(p n+s_{1}\right)+s_{2}\right)+\ldots\right)+s_{k}}^{1 / p^{k}} x^{n}\right]^{p^{k}} \\
& =x^{s}\left[E_{s_{1} \ldots s_{k}}(g)\right]^{p^{k}} \in \Gamma_{K},
\end{aligned}
$$

where $0 \leq s \leq p^{k}-1$ and $E_{i j}=E_{i} \circ E_{j}$, as required.

Note that the set $\Gamma_{K}$ is not closed under the Hadamard product operation, as the following example shows.

EXAmPle 3.6. Let $K=\mathbb{F}_{2}, \theta=\sum_{i=0}^{\infty} \theta_{i} 2^{i}$ and $\phi=\sum_{i=0}^{\infty} \phi_{i} 2^{i}$ be 2-adic integers. Let $\tau=\sum_{i=0}^{\infty} \theta_{i} \phi_{i} 2^{i}$. Then by Lucas' Theorem (see, for example, [2, p. 271]) we have $(1+x)^{\theta} *(1+x)^{\phi}=(1+x)^{\tau}$.

Let $\left(f_{n}\right)_{n \geq 0}$ and $\left(g_{n}\right)_{n \geq 0}$ be two sequences of series in $\mathbb{F}_{2}[[x]]$ and let $f \in \mathbb{F}_{2}[[x]]$ be defined by $f=f_{0}^{2}+x g_{0}^{2}$ and $g_{i}=f_{i+1}^{2}+x g_{i+1}^{2}, i \geq 0$.

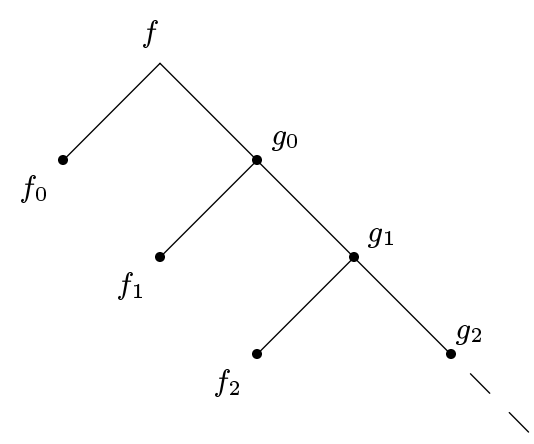

So $f=\sum_{n=0}^{\infty} f_{n}^{2^{n+1}} x^{2^{n}-1}$. Then we have the following lemma. 
LEMma 3.7. $f \in \Gamma_{\mathbb{F}_{2}}$ if and only if $\left\{f_{0}, f_{1}, \ldots\right\} \subseteq \Gamma_{\mathbb{F}_{2}}$ and

$$
\text { tr. } \operatorname{deg}_{\mathbb{F}_{2}(x)} \mathbb{F}_{2}\left(x, f_{0}, f_{1}, \ldots\right)<\infty \text {. }
$$

Proof. See [8].

Consider the set $S=\left\{\theta \in \mathbb{Z}_{2}: \theta_{i}=0\right.$ if $i$ is not a power of 2$\} \cup\{1\}$. Then $S$ is uncountable and hence there exists an infinite sequence $\theta_{(n)}$, $n \geq 0$, of elements of $S$ linearly independent over $\mathbb{Q}$, where $\theta_{(0)}=1$. Let $g_{(n)}=(1+x)^{\theta_{(n)}}, n \geq 1$. Then $g_{(n)}$ are algebraically independent over $\mathbb{F}_{2}(x)$ (see [7]). Now we redefine $\theta_{(0)}=0$ and consider the functions $F$ and $G$ defined as follows:
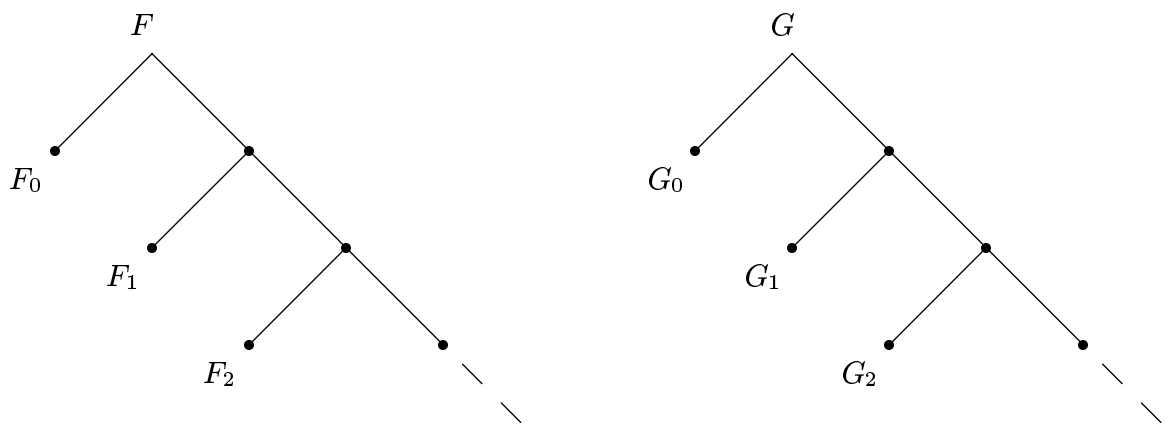

where $F_{n}$ are all equal to $(1+x)^{\theta}, \theta=\sum_{n \geq 0} 2^{2^{n}}$. Also $G_{n}=(1+x)^{\phi \cdot 2^{n}}$, where $\phi=\sum_{i \geq 0} \phi_{i} 2^{i} \in \mathbb{Z}_{2}$ is to be determined.

Note that by Example 2.2, $F$ and $G$ are both in $\Gamma_{\mathbb{F}_{2}}$. Then

$$
F_{n} * G_{n}=(1+x)^{\theta} *(1+x)^{\phi \cdot 2^{n}}=(1+x)^{\lambda^{(n)}},
$$

say, where $\lambda^{(n)}=\sum_{j \geq 0,2^{j} \geq n} \phi_{2^{j}-n} 2^{2^{j}}$ by Lemma 3.6.

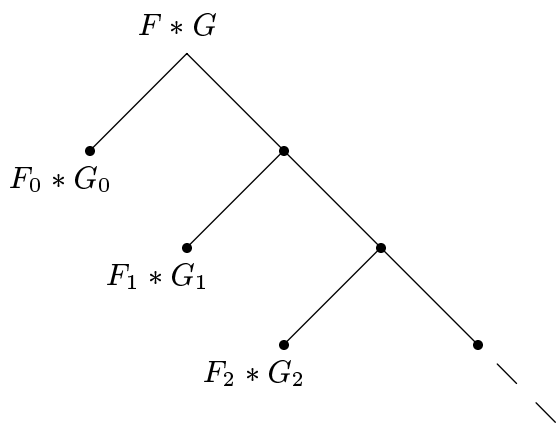

Let $\theta_{(n)}=\sum_{k>0} \theta_{(n)(k)} 2^{2^{k}}$ for $n \geq 0$ as $\theta(n) \in S$. We define $\phi$ as follows: Let $\phi_{0}=0, \phi_{2^{j}-n}=\theta_{(n)(j)}$ for $2^{j}>2 n$. Then

$$
\lambda^{(n)}=\sum_{j \geq 0, n \leq 2^{j} \leq 2 n} \phi_{2^{j}-n} 2^{2^{j}}+\sum_{j \geq 0,2^{j}>2 n} \theta_{(n)(j)} 2^{2^{j}}=\theta_{(n)}+a_{n},
$$


where $a_{n} \in \mathbb{Z}$. Hence $F_{n} * G_{n}=(1+x)^{\lambda^{(n)}}=(1+x)^{a_{n}} g_{(n)}$ for $n \geq 1$ are algebraically independent over $\mathbb{F}_{2}(x)$. Therefore, $F * G \notin \Gamma_{\mathbb{F}_{2}}$ as required.

The classes of $p$-additive and $p$-multiplicative power series are some special types of E-algebraic power series, which are closed under the Hadamard product operation.

Definition. Let $F$ be a field of characteristic $p>0$. We call $f=$ $\sum_{n=0}^{\infty} a_{n} x^{n} \in F[[x]]$ a $p$-multiplicative (respectively, $p$-additive) power series if for all $k, n \in \mathbb{N}$ and $r \in\left[0, p^{k}-1\right]$ we have

$$
\left.a_{p^{k} n+r}=a_{p^{k} n} a_{r} \quad \text { (respectively, } a_{p^{k} n+r}=a_{p^{k} n}+a_{r}\right) .
$$

These series were introduced by Gelfond in [3]. For example, for the $p$-adic integer $\alpha, f_{\alpha}=(1+x)^{\alpha} \in \mathbb{F}_{p}[[x]]$ is a $p$-multiplicative series by Lucas' Theorem, and the series $f=\sum_{n=0}^{\infty} S_{p}(n) x^{n} \in \mathbb{F}_{p}[[x]]$, where $S_{p}(n)$ is the sum of the digits of $n$ in its $p$-adic expansion (see [1]), is a $p$-additive power series.

Proposition 3.8. Any p-multiplicative or p-additive power series in $K[[x]]$ is E-algebraic.

P r o of. Suppose that $f=\sum_{n=0}^{\infty} a_{n} x^{n}$ is $p$-multiplicative. Then by (1),

$$
f=\sum_{i=0}^{p-1} \sum_{n=0}^{\infty} a_{p n+i} x^{p n+i}=\sum_{i=0}^{p-1} a_{i} x^{i} \sum_{n=0}^{\infty} a_{p n} x^{p n}=\sum_{i=0}^{p-1} a_{i} x^{i}\left(E_{0}(f)\right)^{p} .
$$

As $f$ is $p$-multiplicative, $a_{0}=1$, and hence $\left(E_{0}(f)\right)^{p}=f / \sum_{i=0}^{p-1} a_{i} x^{i} \in$ $K(x, f)$ and $\left(E_{i}(f)\right)^{p}=a_{i}\left(E_{0}(f)\right)^{p} \in K(x, f)$ for $0 \leq i \leq p-1$ (see Lemma 2.1). By the same argument we have $E_{0}(f)=\sum_{i=0}^{p-1} a_{p i}^{1 / p} x^{i}\left(E_{00}(f)\right)^{p}$. Hence $\left(E_{00}(f)\right)^{p}=E_{0}(f) / \sum_{i=0}^{p-1} a_{p i}^{1 / p} x^{i}$. Thus

$$
\left(E_{00}(f)\right)^{p^{2}}=\frac{\left(E_{0}(f)\right)^{p}}{\sum_{i=0}^{p-1} a_{p i} x^{p i}}=\frac{f}{\sum_{i=0}^{p-1} a_{i} x^{i} \sum_{i=0}^{p-1} a_{p i} x^{p i}} \in K(x, f)
$$

and similarly $\left(E_{i j}(f)\right)^{p^{2}} \in K(x, f)$ for $0 \leq i, j \leq p-1$. Continuing this process it follows easily that

$$
\left[E_{i_{1} \ldots i_{r}}(f)\right]^{p^{r}}=\frac{c f}{\sum_{i=0}^{p-1} a_{i} x^{i} \sum_{i=0}^{p-1} a_{p i} x^{p i} \ldots \sum_{i=0}^{p-1} a_{i p^{r-1}} x^{i p^{r-1}}},
$$

where $c \in K$ and $E_{i j}=E_{i} \circ E_{j}$. Thus

$$
\left[E_{i_{1} \ldots i_{r}}(f)\right]^{p^{r}} \in K(x, f)
$$

and hence tr. $\operatorname{deg}_{K} K(s, \Omega(f)) \leq 2$. Therefore, $f$ is E-algebraic. 
Now, suppose that $f=\sum_{n=0}^{\infty} a_{n} x^{n}$ is $p$-additive. Then

$$
\begin{aligned}
f & =\sum_{i=0}^{p-1} \sum_{n=0}^{\infty} a_{p n+i} x^{p n+i}=\sum_{i=0}^{p-1} x^{i} \sum_{n=0}^{\infty} a_{p n} x^{p n}+\sum_{i=0}^{p-1} a_{i} x^{i} \sum_{n=0}^{\infty} x^{p n} \\
& =\frac{1-x^{p}}{1-x}\left(E_{0}(f)\right)^{p}+\frac{1}{1-x^{p}} \sum_{i=0}^{p-1} a_{i} x^{i} .
\end{aligned}
$$

Hence

$$
\left(E_{0}(f)\right)^{p}=\frac{1-x}{1-x^{p}}\left[f-\frac{1}{1-x^{p}} \sum_{i=0}^{p-1} a_{i} x^{i}\right] \in K(x, f)
$$

and

$$
\left(E_{i}(f)\right)^{p}=\left(E_{0}(f)\right)^{p}+\frac{a_{i}}{1-x^{p}} \in K(x, f) \quad \text { for } 0 \leq i \leq p-1 .
$$

By the same argument we have

$$
E_{0}(f)=\frac{1-x^{p}}{1-x}\left(E_{00}(f)\right)^{p}+\frac{1}{1-x^{p}} \sum_{i=0}^{p-1} a_{p i}^{1 / p} x^{i}
$$

Hence

$$
\left(E_{00}(f)\right)^{p}=\frac{1-x}{1-x^{p}}\left[E_{0}(f)-\frac{1}{1-x^{p}} \sum_{i=0}^{p-1} a_{i}^{1 / p} x^{i}\right]
$$

Thus

$$
\left(E_{00}(f)\right)^{p^{2}}=\frac{1-x^{p}}{1-x^{p^{2}}}\left[\left(E_{0}(f)\right)^{p}-\frac{1}{1-x^{p^{2}}} \sum_{i=0}^{p-1} a_{p i} x^{p i}\right] \in K(x, f)
$$

since $\left(E_{0}(f)\right)^{p} \in K(x, f)$. Similarly $\left(E_{i j}(f)\right)^{p^{2}} \in K(x, f)$ for $0 \leq i, j \leq p-1$.

By using a simple induction on the order of $E$, one can see that

$$
\left[E_{i_{1} \ldots i_{r}}(f)\right]^{p^{r}} \in K(x, f)
$$

for $i_{j} \in\{0,1, \ldots, p-1\}$ and hence tr. $\operatorname{deg}_{K} K(x, \Omega(f)) \leq 2$. Therefore, $f$ is E-algebraic and the proof is complete.

A similar argument shows that the set of all $p$-multiplicative power series is closed under the Hadamard product operation and hence we have the following.

Corollary 3.9. Let $f, g \in K((x))$ be $p$-multiplicative. Then $f * g$ is E-algebraic.

Proposition 3.10. Let $f, g \in K((x))$ be $p$-multiplicative or $p$-additive. Then $f * g$ is E-algebraic. 
Proof. We just consider the case where $f$ is $p$-additive and $g$ is $p$ multiplicative. Let $f=\sum_{n=0}^{\infty} a_{n} x^{n}$ and $g=\sum_{n=0}^{\infty} b_{n} x^{n}$. Then

$$
\begin{aligned}
h & =f * g=\sum_{n=0}^{\infty} a_{n} b_{n} x^{n}=\sum_{i=0}^{p-1} \sum_{n=0}^{\infty} a_{p n+i} b_{p n+i} x^{p n+i} \\
& =\sum_{i=0}^{p-1} \sum_{n=0}^{\infty}\left[a_{p n}+a_{i}\right] b_{p n} b_{i} x^{p n+i} \\
& =\sum_{i=0}^{p-1} b_{i} x^{i} \sum_{n=0}^{\infty} a_{p n} b_{p n} x^{p n}+\sum_{i=0}^{p-1} a_{i} b_{i} x^{i} \sum_{n=0}^{\infty} b_{p n} x^{p n} \\
& =\sum_{i=0}^{p-1} b_{i} x^{i}\left(E_{0}(h)\right)^{p}+\sum_{i=0}^{p-1} a_{i} b_{i} x^{i}\left(E_{0}(g)\right)^{p} .
\end{aligned}
$$

Since $g$ is $p$-multiplicative, $b_{0} \neq 0$ and hence

$$
\left(E_{0}(h)\right)^{p}=\frac{1}{\sum_{i=0}^{p-1} b_{i} x^{i}}\left[h-\sum_{i=0}^{p-1} a_{i} b_{i} x^{i}\left(E_{0}(g)\right)^{p}\right] \in K(x, g, h),
$$

as $K(x, \Omega(g), h)=K(x, g, h)$. Similarly, we get

$$
\left(E_{i}(h)\right)^{p}=b_{i}\left[\left(E_{0}(h)\right)^{p} a_{i}\left(E_{0}(g)\right)^{p}\right] \in K(x, g, h)
$$

for $0 \leq i \leq p-1$. Now by a similar argument we get

$$
\left(E_{00}(h)\right)^{p}=\frac{1}{\sum_{i=0}^{p-1} b_{p i}^{1 / p} x^{i}}\left[E_{0}(h)-\sum_{i=0}^{p-1} a_{p i}^{1 / p} b_{p i}^{1 / p} x^{i}\left(E_{00}(g)\right)^{p}\right] .
$$

Thus $\left(E_{00}(h)\right)^{p^{2}}$ and similarly $\left(E_{i j}(h)\right)^{p^{2}}$ are in $K(x, g, h)$ for $0 \leq i, j \leq p-1$. By using a simple induction on the order of $E$, one can see that

$$
\left[E_{i_{1} \ldots i_{r}}(h)\right]^{p^{r}} \in K(x, g, h)
$$

for $i_{j} \in\{0,1, \ldots, p-1\}$ and hence tr. $\operatorname{deg}_{\cdot K} K(x, \Omega(h)) \leq 3$. Therefore, $h=f * g$ is E-algebraic.

4. Diagonals of D-algebraic and E-algebraic power series. The notions of D-algebraicity and E-algebraicity can be generalised to the case of several variables. In this section we shall study the diagonal of such series.

For a power series

$$
f=\sum_{n_{j} \geq 0} a_{n_{1} \ldots n_{k}} x_{1}^{n_{1}} \ldots x_{k}^{n_{k}}
$$

the series

$$
D(f)=\sum_{n=0}^{\infty} a_{n \ldots n} t^{n}, \quad \text { where } \quad t=x_{1} \ldots x_{k}
$$


is called the diagonal of $f$. Although taking the diagonal of $f$ just amounts to taking the Hadamard product of $f$ with the rational function $g=$ $1 /\left(1-x_{1} \ldots x_{k}\right)$, the following example shows that Theorem 3.2 is not true for the case of several variables. That is, the diagonal of a D-algebraic power series need not be D-algebraic.

ExAmPle 4.1. Suppose that

$$
f(x, y)=\frac{e^{y}}{2}[\theta(x)+1]
$$

where $\theta(x)$ is the theta function. Let

$$
f_{1}(x)=\frac{1}{2}[\theta(x)+1]=\sum_{n=0}^{\infty} x^{n^{2}} \quad \text { and } \quad f_{2}(y)=e^{y} .
$$

Then $f_{1}$ and $f_{2}$ are both D-algebraic. Now,

$$
f_{1}(x) f_{2}(y)=f(x, y)=\left(\sum_{n=0}^{\infty} x^{n^{2}}\right)\left(\sum_{k=0}^{\infty} \frac{1}{k !} y^{k}\right)
$$

and hence $D(f)=\sum_{n \geq 0}\left(1 / n !^{2}\right) t^{2^{n}}$, which is not D-algebraic (see [4]). Therefore, we have the following

Corollary 4.2. Suppose that $k>1$ and $f, g \in L\left[\left[x_{1}, \ldots, x_{k}\right]\right]$. If $f$ is rational and $g$ is D-algebraic, then $f * g$ is not necessarily D-algebraic.

We shall show below that the diagonal of an E-algebraic power series in several variables is not necessarily E-algebraic either and hence Theorem 3.3 is not true for the case of several variables.

Let $F$ and $G$ be the functions of Example 3.6. Consider $F(x)$ and $G(y)$ as the elements of $\mathbb{F}_{2}[[x, y]]$. Then, as $\Gamma_{\mathbb{F}_{2}}$ is a field (see $[8]$ ), $F(x) G(y)$ is E-algebraic. Let $F(x)=\sum_{n=0}^{\infty} a_{n} x^{n}$ and $G(y)=\sum_{m=0}^{\infty} b_{m} y^{m}$. Then $F(x) G(y)=\sum_{n=0}^{\infty} \sum_{m=0}^{\infty} a_{n} b_{m} x^{n} y^{m}$. So $D(F G)=\sum_{n=0}^{\infty} a_{n} b_{n} t^{n}$, where $t=x y$. However, $D(F G)=F(x) * G(x)$. Therefore, we have the following

Corollary 4.3. Suppose that $k>1$ and $f, g \in K\left[\left[x_{1}, \ldots, x_{k}\right]\right]$. If $f$ is rational and $g$ is E-algebraic, then $f * g$ is not necessarily E-algebraic.

The only remaining cases in studying the Hadamard products of Dalgebraic and E-algebraic power series are the following questions for series in one variable, which seem to be open.

Is the Hadamard product of an algebraic power series and a D-algebraic (respectively, E-algebraic) power series D-algebraic (respectively, Ealgebraic)?

Acknowledgements. I would like to thank the Research Council of Shiraz University. I would also like to thank Professor L. Lipshitz for bringing to my attention Example 4.1 and the referees for their comments. 


\section{References}

[1] J.-P. Allouche, Somme des chiffres et transcendance, Bull. Soc. Math. France 110 (1982), 279-285.

[2] L. E. Dickson, History of the Theory of Numbers, Vol. 1, Carnegie Institution of Washington, Washington, 1919.

[3] A. O. Gelfond, Sur les nombres qui ont des propriétés additives et multiplicatives données, Acta Arith. 13 (1967), 259-265.

[4] L. Lipshitz and L. Rubel, A gap theorem for power series solutions of algebraic differential equations, Amer. J. Math. 108 (1982), 1193-1214.

[5] M. Mendès-France and A. J. van der Poorten, Automata and the arithmetic of formal power series, Acta Arith. 46 (1986), 211-214.

[6] H. Sharif, Children products of formal power series, Math. Japon. 38 (1993), 319324 .

[7] —, Algebraic independence of certain formal power series, J. Sci. I.R.I. Iran 2 (1991), $50-55$.

[8] - E-algebraic functions over fields of positive characteristic-an analogue of differentially algebraic functions, J. Algebra 207 (1998), 355-366.

[9] H. Sharif and C. F. Woodcock, Algebraic functions over a field of positive characteristic and Hadamard products, J. London Math. Soc. 37 (1988), 395-403.

[10] R. P. Stanley, Differentiably finite power series, European J. Combin. 1 (1980), $175-188$.

Department of Mathematics

Shiraz University

Shiraz 71454, Iran

E-mail: sharif@sun01.susc.ac.ir

Received on 7.1 .1998

and in revised form on 9.3.1999 